\title{
Wakefields in SLAC linac collimators
}

\author{
A. Novokhatski, ${ }^{*}$ F.-J. Decker, H. Smith, and M. Sullivan \\ SLAC National Accelerator Laboratory, Stanford University, Menlo Park, California 94025, USA
}

(Received 18 June 2014; published 2 December 2014)

\begin{abstract}
When a beam travels near collimator jaws, it gets an energy loss and a transverse kick due to the backreaction of the beam field diffracted from the jaws. The effect becomes very important for an intense short bunch when a tight collimation of the background beam halo is required. In the Linac Coherent Light Source at SLAC a collimation system is used to protect the undulators from radiation due to particles in the beam halo. The halo is most likely formed from gun dark current or dark current in some of the accelerating sections. However, collimators are also responsible for the generation of wake fields. The wake field effect from the collimators not only brings an additional energy jitter and change in the trajectory of the beam, but it also rotates the beam on the phase plane, which consequently leads to a degradation of the performance of the Free Electron Laser at the Linac Coherent Light Source. In this paper, we describe a model of the wake field radiation in the SLAC linac collimators. We use the results of a numerical simulation to illustrate the model. Based on the model, we derive simple formulas for the bunch energy loss and the average kick. We also present results from experimental measurements that confirm our model.
\end{abstract}

DOI: 10.1103/PhysRevSTAB.17.124401

PACS numbers: 41.75.Ht, 41.85.Ja, 29.20.Ej

\section{INTRODUCTION}

The electron beam collimators in the LCLS must remove the halo particles in the beam before they affect and eventually degrade the very precise fields of the permanent magnet undulators [1]. However, collimators also cut the electromagnetic field of the electron bunch and produce (in this way) freely propagating fields which are wake fields. The wake field effect of collimators with small apertures on the transverse beam dynamics was observed during the operation of the Stanford Linear Collider (SLC) [2]. Several attempts were made to estimate and to measure the effect of collimator transverse wake fields [2-6]. Special measurements have been made for longitudinally tapered collimators, designed for the applications in future linear colliders. Detailed analysis of these measurements and analytical results can be found in Ref. [6]. Different formulas have been suggested to describe the collimator kick, but unfortunately they do not explain the experimental results very well in many cases.

The problem of wake fields excited by collimators becomes more important for linac operation and $\mathrm{x}$-ray production at the LCLS. The backward reaction of the wake field from the collimators on the beam brings an additional energy jitter and a change to the trajectory of the beam. It also leads to a degradation of the FEL performance at the LCLS. This is because of the special character of the wake

\footnotetext{
*novo@slac.stanford.edu

Published by the American Physical Society under the terms of the Creative Commons Attribution 3.0 License. Further distribution of this work must maintain attribution to the author $(s)$ and the published article's title, journal citation, and DOI.
}

fields: the response reaction depends on the longitudinal position of the particles in the bunch. The "head" of the bunch is not deflected at all, but the "tail" gets the maximum deflection force. This kind of kick leads to the bunch being geometrically tilted. Because the tail of the bunch may oscillate in the lattice, the orientation of the bunch in space will oscillate too. Effectively, the transverse projected emittance is increased and the FEL performance is degraded.

We suggest that because of the rectangular shape of the collimator jaw, a geometrical diffraction model may be used to calculate the longitudinal and transverse wake fields excited by a short bunch. This approach is described in Ref. [7]. Because it is difficult to find this reference, we will briefly explain the model following the description of the SLAC linac collimator below.

\section{SLAC LINAC COLLIMATOR}

Nine adjustable beam collimators are used in the LCLS operation, mainly accomplished in two main sections: at the end of the SLAC linac and in the region from the linac to the undulators (LTU). Each collimator is composed of horizontal and vertical pairs of rectangular collimator jaws. A sketch of a vertical collimator is shown in Fig. 1. The geometry of a collimator assembly is very complicated because each jaw is independently and remotely adjustable and can completely shadow the beam path. In the center of Fig. 1, we can see two longitudinal jaws with bellows. The beam moves in a horizontal direction, and part of the beam (beam halo) can be dissipated in jaws. The length of a jaw is not enough to fully capture the high-energy beam $(4-14 \mathrm{GeV})$.

A collimator is essentially a kind of assembly of $\mathrm{rf}$ cavities coupled to the beam. The bellows with a chamber 


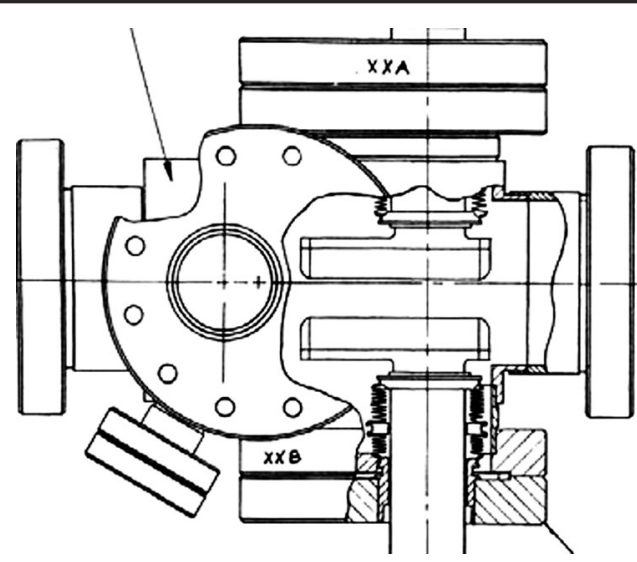

FIG. 1. Sketch of a SLAC linac collimator. The dash-dot line can be a beam trajectory when the beam goes close to the upper jaw.

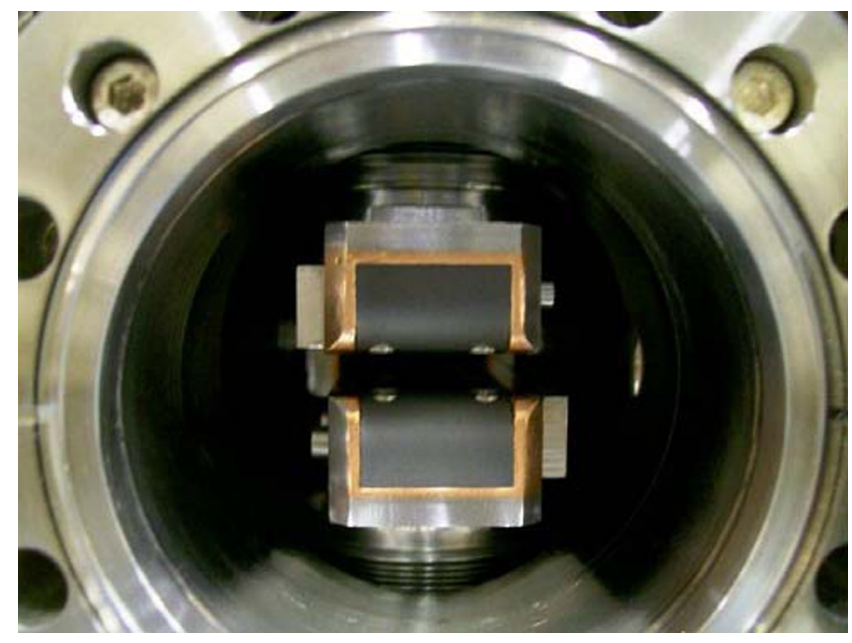

FIG. 2. A vertical collimator.

form two quarter-wave coaxial cavities. There are several trapped rf modes inside the collimator volume. In a multibunch operation, some energy is deposited in this region. One jaw can be in a position that is too close to the beam path while the other jaw has been moved out. The jaws have a titanium alloy body with a slightly curved face (10-m radius) and a titanium-nitride jaw surface for improved conductivity and survivability against beam hits [8]. Currently the gap between jaws is kept approximately $\pm 1.6 \mathrm{~mm}$ in all collimators. However, the spontaneous beam halo requires smaller gaps. A photo of a vertical collimator is shown in Fig. 2. We can see how the jaws together with the bellows strongly disturb the smooth inner surface of the beam pipe.

\section{GEOMETRIC OPTICS OF THE WAKE FIELDS}

As an introduction let us consider the dynamics of the radiation fields when a bunch travels through a hole in a metal diaphragm placed in a pipe. Figure 3 illustrates the picture of the force lines of an electric field of a bunch at

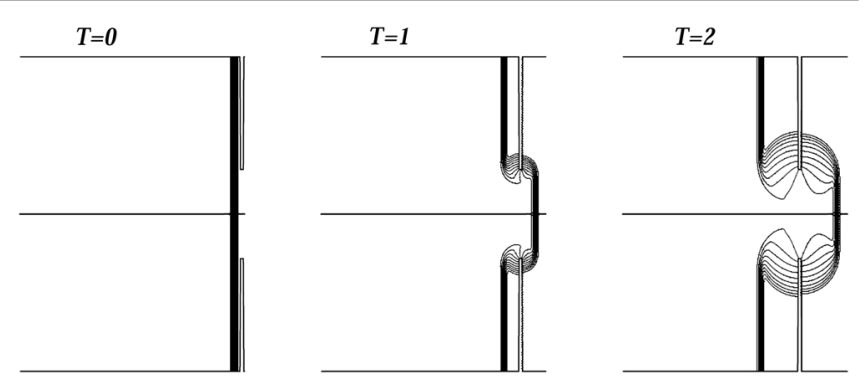

FIG. 3. Plot of electric force lines for three time steps: a bunch approaches the diaphragm $(T=0)$, the bunch passes the diagram $(T=1)$ and the bunch is now past the diaphragm $(T=2)$.

various moments: the bunch before the diaphragm, passing the diaphragm, and beyond the diaphragm.

When the bunch approaches the diaphragm it carries its "own" field, which has only transverse components in the ultrarelativistic case ( $T=0$ in Fig. 3). Some part of the "self" field is cut off by the diaphragm and reflected back into the beam pipe ( $T=1$ in Fig. 3). Beyond the diaphragm, the bunch forms its own field, giving off a fraction of its kinetic energy to the field energy ( $T=2$ in Fig. 3).

According to the law of superposition of the electromagnetic fields we can separate the bunch self field from the total field. In the case of an infinitely thin diaphragm, the field behind the diaphragm can be represented as a sum of the bunch self field and the "cutoff" field; the latter being the same as to the left of the diaphragm but mirror reflected. Figure 4 illustrates this concept.

The cutoff and the "mirror-reflected" fields are separated from a bunch and propagate independently. We usually call these fields wake fields. It can be seen in Fig. 4, that the energy of the cutoff field determines the backward-emitted energy. The mirror-reflected field is propagating in the forward direction and chasing the bunch. The energy of the mirror-reflected field is almost the same as the energy of the cutoff field for an infinitely thin diaphragm. So the total energy loss will be equal to the double energy of the cutoff field. In the calculation of the energy of the cutoff field we need to also include the energy of the magnetic component of the bunch field

$$
\Delta W=\int d z \int_{S}\left(\varepsilon_{0} E_{b}^{2}+\mu_{0} H_{b}^{2}\right) d s
$$
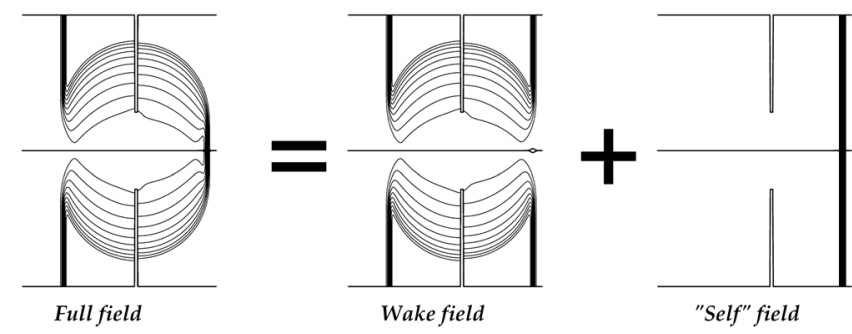

FIG. 4. Total field as a sum of wake fields and a bunch self field. 
Fortunately, this is easy to do because in the ultrarelativistic case the energy of the electric component is the same as the energy of the magnetic component of the bunch field. The averaged potential for a bunch with a total charge $Q$ will be

$$
\Delta E=\frac{\Delta W}{Q} .
$$

With this concept of the radiation process one can obtain estimates for the energy loss of a bunch in some simple cases. For example, the energy, emitted by a pointlike charged particle passing through a hole in an infinite metal screen, will be proportional to the energy of a particle and inversely proportional to the radius of the hole in a diaphragm

$$
\Delta E \sim \gamma \frac{Q}{2 \pi \varepsilon_{0} a} .
$$

$Q$ is the total bunch charge, $\gamma$ is a relativistic factor, $\varepsilon_{0}$ is a dielectric constant of free space, and $a$ is a radius of the hole in a diaphragm. It is necessary to emphasize that the radiation energy increase is linear with the particle energy of the bunch. Formula (3), derived in this very simple way, is in good agreement with the results of more complicated analytical calculations, which were carried out later [9].

If a bunch is not a pointlike particle but has a finite length $l_{b}$, then the energy loss is logarithmic with the particle energy

$$
\Delta E \sim \frac{Q}{\pi \varepsilon_{0} l_{b}} \ln \gamma \frac{l_{b}}{a},
$$

where $l_{b}$ is the total bunch length.

If a diaphragm is placed inside a vacuum chamber and the transverse size of the chamber $b$ meets the condition that $b<\gamma l_{b}$, then the energy loss does not depend upon the particle energy

$$
\Delta E=\frac{Q}{\pi \varepsilon_{0} l_{b}} \ln \frac{b}{a} .
$$

The energy loss then depends mainly upon the bunch length (inversely proportional) and only logarithmically upon the geometrical parameters of the beam chamber and the diaphragm. For a bunch with a Gaussian charge distribution and a bunch length of $\sigma$, the equivalent total bunch length is $l_{b}=2 \sqrt{\pi} \sigma$.

The same formula in Eq. (4) but with a coefficient of one-half gives us the energy loss for the case when a bunch crosses a region with a sharp change in the transverse cross section while moving from a chamber with a smaller size to a chamber with a larger size. The energy loss describes the kinetic energy that is used by the bunch for the formation of the bunch self field in the larger chamber. For the inverse problem (i.e., the bunch goes from a chamber of larger size to a chamber with a smaller size) the above relation with one-half determines the energy of the reflected back field when the kinetic energy of the particles in the bunch remains nearly the same. It was found that this approach is in very good agreement with a more detailed analysis of the azimuthal symmetry structures. Reference [10] describes an approximate analytical expression of the Green's function for the wake fields which corresponds to the case when a disk with a hole is installed in a cylindrical tube. This Green's function is

$$
\begin{aligned}
G(s) & =\delta(s) \frac{c Z_{0}}{\pi} \ln \frac{b}{a}-\frac{1}{a \pi} F_{0}\left(\frac{s}{a}\right), \\
F_{0}(x) & =\left\{\begin{array}{ll}
\frac{2}{x} \arcsin \left(\frac{2}{x}\right) & \text { for } x \leq 2 \\
\frac{\pi}{x} & \text { for } x>2
\end{array} .\right.
\end{aligned}
$$

The main part of the Green's function is a delta function which means that an energy loss inside the bunch is proportional to the bunch charge density. This gives us a simple way to calculate the main part of the wake field potential of a bunch with a very complicated charge shape. A full description is given by the formula below for any bunch charge density $q(s)$

$$
W(s)=q(s) \frac{c Z_{0}}{\pi} \ln \frac{b}{a}-\frac{1}{a \pi} \int_{0}^{\infty} q\left(s^{\prime}\right) F_{0}\left(\frac{s-s^{\prime}}{a}\right) d s^{\prime} .
$$

In Fig. 5, we show the wake potential of a double-horn bunch calculated for a cylindrical model of the SLAC collimator. The dashed line shows the bunch charge density distribution.

Recall that the bunch energy loss can be calculated using a so-called loss factor and the total bunch charge

$$
\delta E=\kappa_{\text {loss }} \cdot Q .
$$

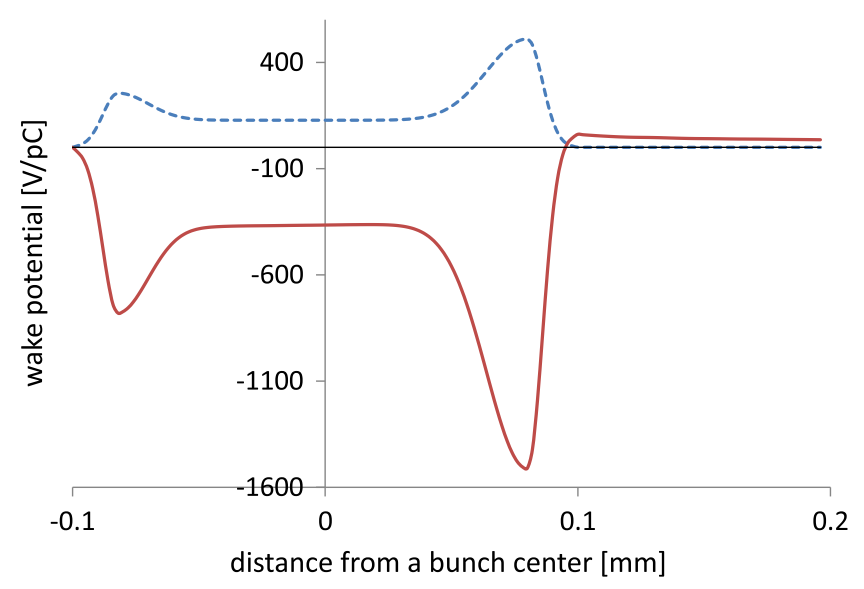

FIG. 5. Wake field potential of a collimator chamber (cylindrical model) excited by a double-horn bunch. The dashed line shows the bunch charge distribution. 
The loss factor is equal to the energy loss of a unit charge. To calculate a loss factor we integrate the wake potential with the bunch charge density

$$
\kappa_{\mathrm{loss}}=\frac{1}{Q} \int_{-\infty}^{\infty} W(s) q(s) d s .
$$

Using this loss factor, we may also calculate the average power of the energy loss, which must be the same as the power of the field generated by a train of bunches with a bunch spacing $\tau$ and an average beam current $I_{a v}$

$$
P=\kappa_{\operatorname{loss}} I_{a v}^{2} \tau .
$$

In order to study the wake field dynamics and main properties of the fields in the collimator region we carried out computer simulations for a cylindrical model of a collimator using the numerical code NOVO [11].

\section{WAKE FIELD DYNAMICS IN A COLLIMATOR}

The cylindrical model of the SLAC linac collimator is shown in Fig. 6. It consists of a collimator cavity and a symmetrical jaw. The diameter of a hole inside a cylindrical jaw is equal to the gap size of $3.2 \mathrm{~mm}$. A short bunch goes from the left side of the picture to the right. The plot of the electric force lines (or field lines) is shown at the moment when a bunch has passed through the jaw. At this time a bunch is at the right side of the picture. The green lines show the electric force lines, which have a positive longitudinal component. They show the distribution of the accelerating forces. The blue lines show the electric force lines, which have a negative longitudinal component. They show the distribution of decelerating forces. It can be seen from the shape of the force lines that they are trying to "stop" a bunch. In the symmetrical case, this force has only a longitudinal component, which decelerates the beam.

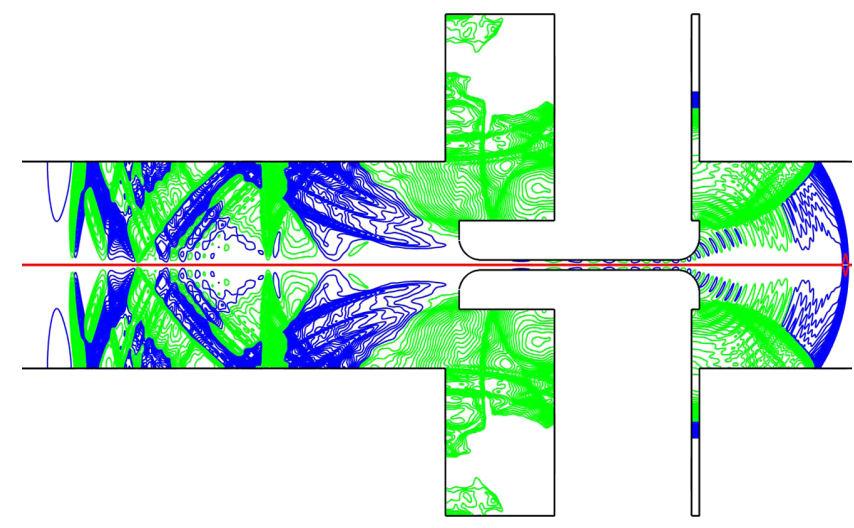

FIG. 6. Cylindrical model of the SLAC linac collimator with electric field lines (blue and green lines) of the wake fields, excited by a short bunch. Red line shows the bunch charge distribution.
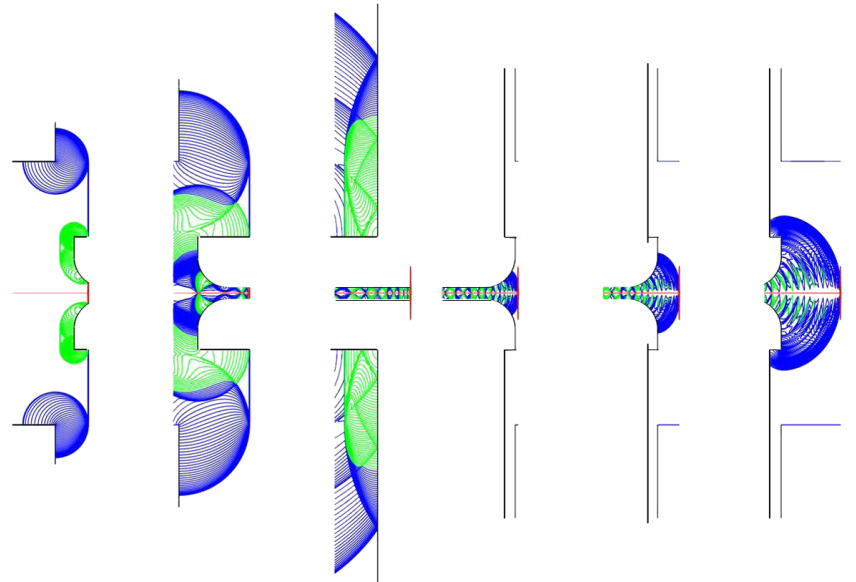

FIG. 7. Dynamics of wake fields, excited by a short bunch passing through a collimator. Blue and green lines show the electric force lines. Red vertical line shows the position of the bunch.

This shows how the bunch particles lose energy. If a bunch is closer to one of the jaws then this force loses symmetry and gets a transverse component. This transverse component kicks the bunch in the direction of the jaw.

The part of the bunch field, which is initially deflected by a jaw, reflects also from the walls of the collimator chamber. After multiple reflections, only trapped rf modes will stay inside the collimator chamber. These rf modes will be eventually absorbed by the stainless steel surface of the walls of the collimator chamber and bellows. The part of the field that propagates between the jaws (or inside a symmetrical jaw) contains very high frequency waves. We can see these waves propagating out of the jaws at an angle. We assume that this effect is due to the specially rounded shape of the jaws. The detailed plot of the wake field radiation at different positions of a $50-\mu \mathrm{m}$ bunch in a collimator chamber is shown in Fig. 7. Initially, the radiation starts from the corner of the collimator chamber, then from the edge of the jaw. Because of the special shape of the jaw the high frequency waves easily enter the hole in the jaw and follow a bunch inside the jaw. At the exit of jaws, they easily escape. The radiation coming from the exit edge of the jaw is very similar to the radiation of waves from a horn antenna. This $\mathrm{THz}$ radiation can be seen outside of the beam pipe as the SLAC collimator chamber has an optical window watching this place (unfortunately not shown in Fig. 1).

We have calculated a very long wake potential of the collimator chamber excited by a $50-\mu \mathrm{m}$ Gaussian bunch. The first part of this wake potential is shown at Fig. 8. We can see that the main part of the potential is near the bunch and has the shape of the bunch charge distribution. This confirms that the Green's function of the collimator wake fields is really a delta function. More than that, this part is very well described by expression (6). After the bunch, the wake potential contains a lot of spikes. To see them more 


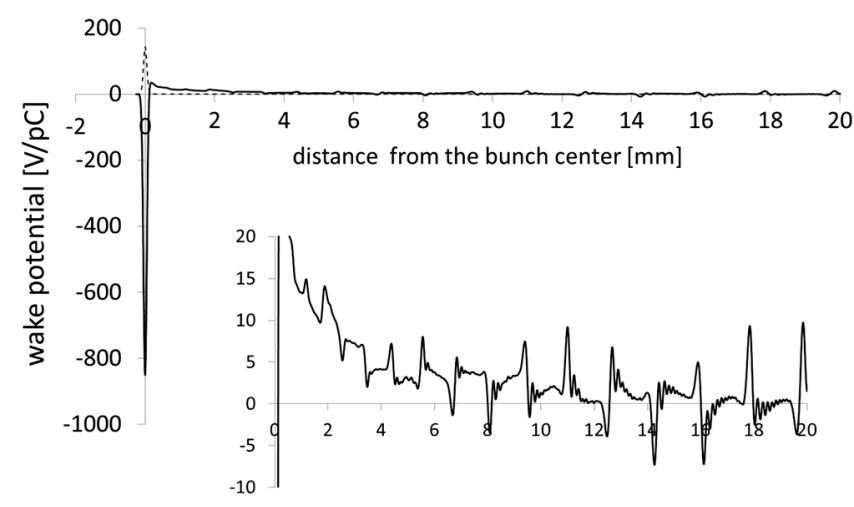

FIG. 8. Wake field potential of a collimator chamber, excited by a $50 \mu \mathrm{m}$ bunch and an enlarged inset showing the detailed structure of the high frequency radiation. The dashed line in the upper graph at $\mathrm{x} \sim 0$ shows the bunch charge distribution.

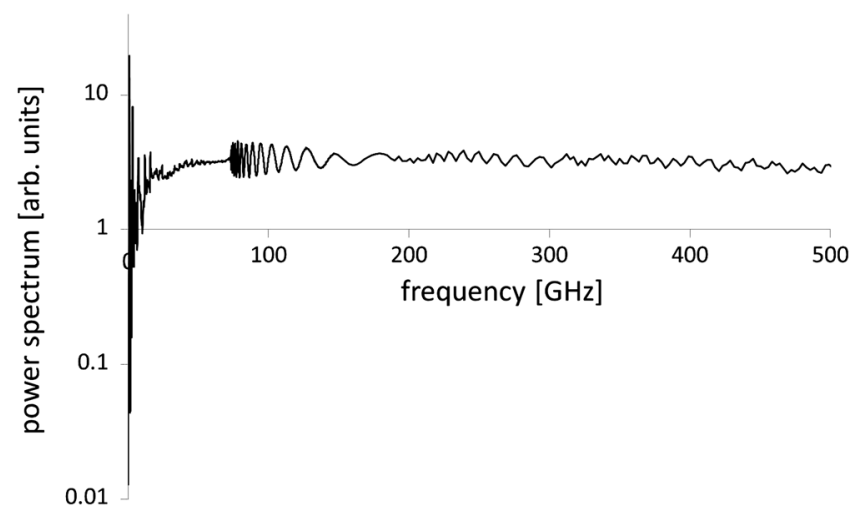

FIG. 9. Power spectrum of the wake potential of the collimator chamber, excited by a $50 \mu \mathrm{m}$ bunch.

clearly in Fig. 8, we include a part of the graph with an enlarged vertical scale. The distances between the spikes are correlated with the size of the hole in the jaw. Between the spikes, we can see very high frequency radiation in the $\mathrm{THz}$ range.

We carried out a Fourier spectrum analysis of this wake field potential. The result is shown in Fig. 9 in the form of a power spectrum. In general, the spectrum is flat, which corresponds to the delta function of the Green's function of the wake fields. There are several trapped modes below the cutof frequency of $3.3 \mathrm{GHz}$ and some very interesting behavior in the region of $80-100 \mathrm{GHz}$.

\section{ESTIMATE OF THE ENERGY LOSS}

We may assume that the Green's function in the form of a delta function may describe a more complicated geometry. Let us rewrite expression (2) in a more general form

$$
\Delta E=\frac{I_{b} Z_{0}}{\pi} K
$$

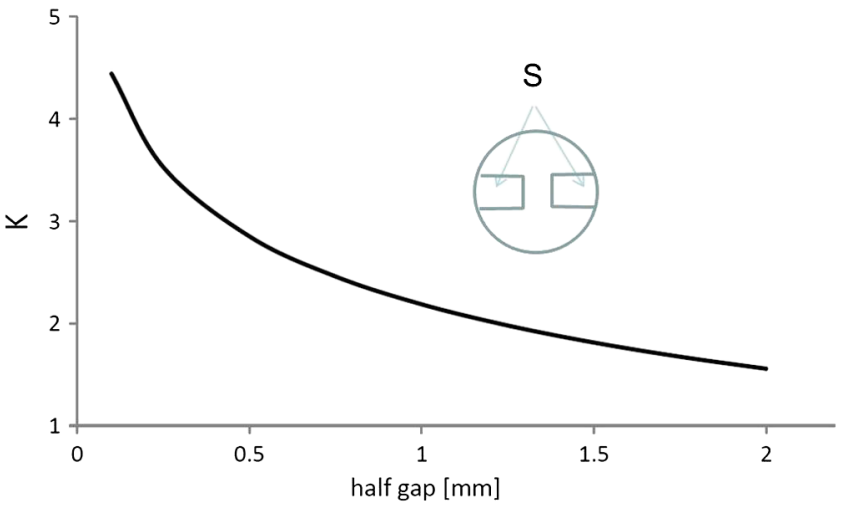

FIG. 10. Parameter $K$ for the energy loss calculation as a function of the distance between jaws.

where $I_{b}=\frac{Q}{c l_{b}}$ is a bunch peak current, $Z_{0}$ is the impedance of free space, $Z_{0}=120 \pi[\mathrm{Ohm}]$, and $K$ is a dimensionless parameter, which is calculated according to the following formula:

$$
K=\frac{1}{2 \pi} \iint_{S} \frac{d x d y}{x^{2}+y^{2}} .
$$

This is an integral over the cross section $(S)$ of a nonhomogeneous element, which is placed inside a vacuum chamber. In our case this element is a set of two collimator jaws.

We have calculated the parameter $K$ for the SLAC collimator for different values of the gap (Fig. 10). We define a gap size as the distance between two jaws.

For large gaps the parameter $K$ varies slowly. A quick estimate gives its value to be around one. For small gaps $K$ approaches infinity and can be approximated by an inverse function of the gap size plus a constant. In Fig. 11 we also show bunch energy loss as a function of the gap size for different bunch currents.

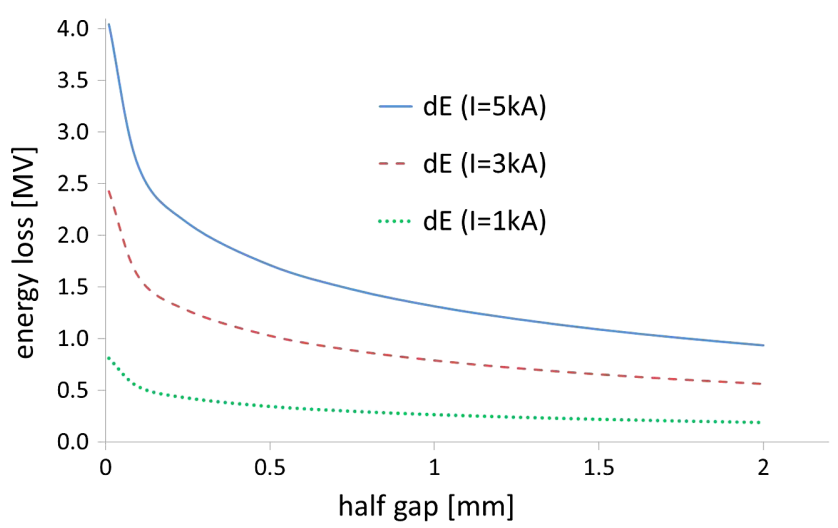

FIG. 11. Bunch energy loss as a function of the gap between jaws for different bunch currents: $1 \mathrm{kA}$ : dotted line; $3 \mathrm{kA}$ : dashed line; $5 \mathrm{kA}$ : solid line. 
Usually, the LCLS operates at a bunch current of $5 \mathrm{kA}$ and a bunch charge of $150-250 \mathrm{pC}$ with a repetition rate of up to $120 \mathrm{~Hz}$. The average beam current may reach $30 \mathrm{nA}$. Figure 11 tells us that the particle energy loss of 1.5-2 MeV may be reached at gap sizes of 1-2 $\mathrm{mm}$. It is important to note that because of the special type of wake field excited in the collimator chamber all particles will get approximately the same energy loss; at least in the main part of a bunch as can be seen at Fig. 5 for a two-horn distribution. The feedback system, which controls the beam energy at LCLS, will compensate this energy loss and there will be no strong effect on FEL operation. The power loss from one collimator is very small; it is less than $0.02 \mathrm{~W}$.

\section{A TRANVERSE KICK FROM A COLLIMATOR JAW}

As we discussed before, in the case of a small gap, the loss factor of short bunches is inversely proportional to the distance between jaws. We may assume that it will be the same dependence if we have only one jaw, then the equivalent distance will be the distance between a beam and a jaw. The same dependence must be true for the transverse wake fields. We suggest the following estimate for a kick of a particle that has a longitudinal position $s$ in a bunch,

$$
g(s)=\frac{Z_{0}}{4 \pi} I_{b} \frac{s}{\delta} .
$$

$\delta$ is the transverse distance between a bunch and a collimator jaw. The average bunch kick then is

$$
g_{a v}=\frac{1}{8 \pi \varepsilon_{0}} \frac{Q}{\delta} .
$$

Opposite to the energy loss, which is proportional to the bunch current, the average kick is determined by the bunch charge and how close a beam is to the edge of a collimator jaw. The nonlinear behavior of the kick leads immediately to emittance growth if a bunch travels very close to a collimator jaw edge. However even a linear kick may increase an effective or projected emittance because a bunch "head" and a bunch "tail" will get a different kick. A head will get nothing, but a tail will get a maximum kick.

\section{A DIPOLE KICK FROM A COLLIMATOR WITH TWO JAWS}

If we know a kick from one jaw, we can calculate a kick from a collimator with two jaws. Each jaw attracts the beam and the total kick must be the sum of the two kicks,

$$
g_{a v}=\frac{Q}{8 \pi \varepsilon_{0}}\left(\frac{1}{\delta_{1}}-\frac{1}{\delta_{2}}\right)
$$

where $\delta_{1}$ and $\delta_{2}$ denote the displacement from the two jaws. If a bunch is near a symmetry plane between two jaws and has a small displacement $\Delta x$, then we get a dipole kick

$$
g_{a v} \approx \frac{Q}{4 \pi \varepsilon_{0}} \frac{\Delta x}{a^{2}} \quad \Delta x \ll a .
$$

Here we assume that the distance between the two jaws is $2 a$. This formula gives the same dipole kick we calculate using the Green's function for the azimuthal harmonic $m=1$ in Ref. [10]. In addition, this formula also can be found in Refs. [12], [4], and [3], which are placed in historical sequence. The average kick is proportional to the displacement of a bunch from the symmetry plane and inversely proportional to the square of the distance between the jaws, contrary to the theoretical model in Ref. [2]. That model predicts a kick inversely proportional to the bunch length and the distance between jaws. This model did not get agreement with experimental results.

\section{MEASUREMENTS AND DATA ANALYSIS}

The SLAC collimators are installed mainly at the end of the linac and in the LTU (linac to undulator) beam line. The beam line regions that contain the collimators are detailed in [1]. We use upstream and downstream beam position monitors (BPMs) to determine the incoming and outgoing trajectory of the beam. The measurement of the beam positions at the locations of these BPMs allow us to measure the kick angle imparted by the transverse wake fields. The BPMs also measure the bunch charge. We use this information to determine when a jaw edge starts to intercept the beam.

The initial beam trajectory corresponds to normal LCLS operation when the beam is centered in the collimators by the feedbacks. We record BPM data sets for each position of the collimator jaw (20 machine pulses). For each measurement only one jaw is moved in the direction towards the collimator center, the other jaw is kept far away from the beam. We change the jaw position by steps of $0.05 \mathrm{~mm}$ or less. Measurements of the beam kick due to the collimator wake fields were made with a beam energy of $11.5 \mathrm{GeV}$ and a bunch charge of $150 \mathrm{pC}$.

For every jaw position we averaged all 20 machine pulses removing any failed pulse or BPM malfunction. We also calculated a ratio of a bunch charge change before and after the collimator in use with the BPM data. We show this ratio for one of the vertical collimators in Fig. 12. The red circles correspond to the measured values. In this case we moved a vertical jaw down. As the collimator jaw starts to tackle the beam, the ratio goes down. When the bunch charge loss reaches $50 \%$ we conclude the collimator jaw edge is in the center of the bunch. We have to note that the measured position of the beam may not be in the geometrical center of the collimator. There can be some displacement. If we assume that the transverse distribution of the 


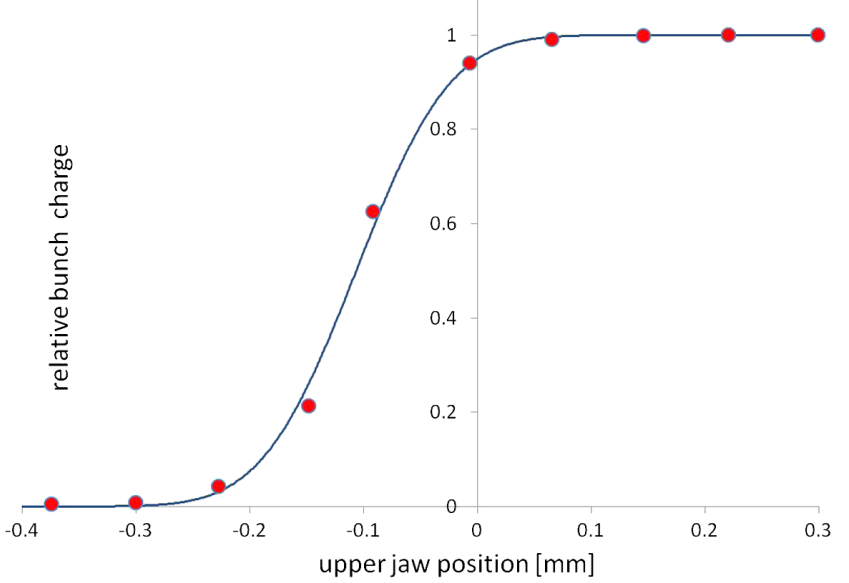

FIG. 12. Ratio of an averaged bunch charge before and after the collimator as a function of the collimator jaw position (red circles) and the analytical approximation (black solid line). An upper jaw is moving down in this case.

bunch charge has a Gaussian shape then we can approximate the measured data by the error function using the displacement and the bunch size as varying parameters. In this way, we can determine the displacement of a bunch relative to a collimator jaw and the bunch size. The black solid line in Fig. 12 shows this approximation. We found that the displacement is $106 \mu \mathrm{m}$ for this collimator and the vertical bunch size is equal to $65 \mu \mathrm{m}$. Measurement with a horizontal collimator showed that the horizontal beam size is the same as the vertical of $65 \mu \mathrm{m}$.

For the transverse kick analysis we choose only those positions of a jaw when the ratio of a bunch charge has not changed much. Our first goal was to determine the direction of the kick induced by the wake fields. We take a reference trajectory along the linac, LTU and undulator regions when a jaw is far away from the beam. We then measure the trajectory when a jaw is close to the beam and take the difference between these trajectories.

Figure 13 shows the difference of the horizontal projections of the trajectories downstream of a horizontal collimator when the left jaw is moved towards the beam.

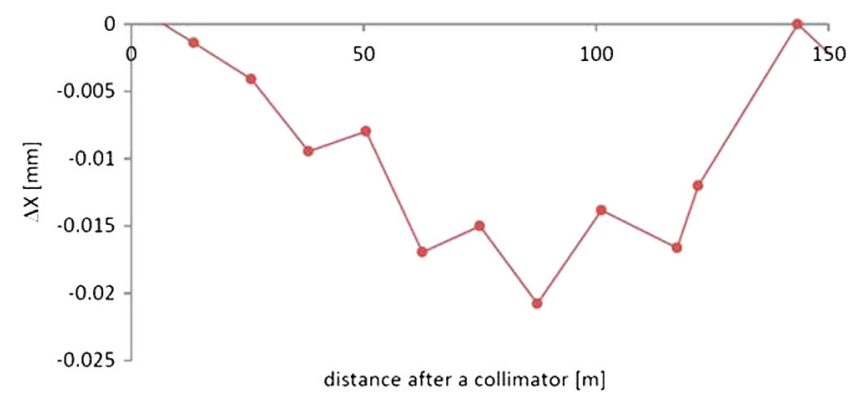

FIG. 13. Difference of the horizontal projections of the beam trajectories downstream of a horizontal collimator when the left jaw is moved towards the beam.

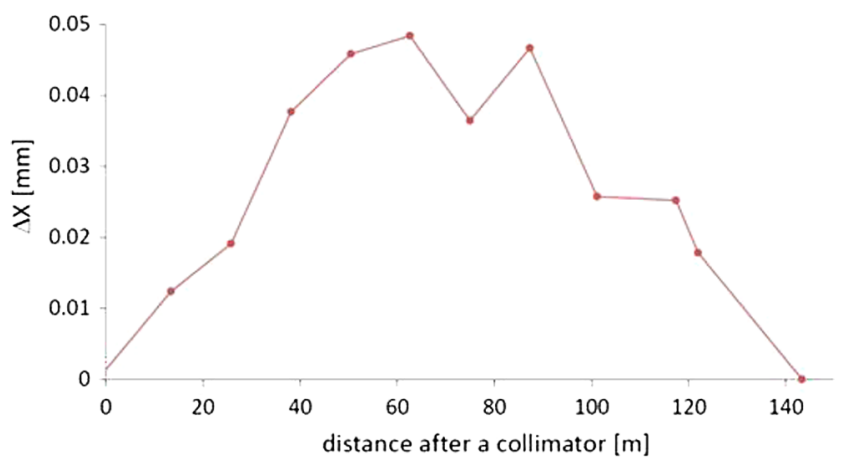

FIG. 14. Difference between the horizontal projections of the beam trajectories downstream of a horizontal collimator when the right jaw is moved towards the beam.

We can see that the beam gets a negative kick. The apparent oscillations in the data is the result of a beta oscillation due to the optics in this region.

When we move the right jaw of the same collimator towards the beam, the beam gets a positive kick, as can be seen in Fig. 14. Here the displacement is larger compared to the left jaw because we moved the right jaw closer to the beam.

We can see that in both cases the beam gets a kick in the direction of the close jaw. In some sense, we can say that a jaw "attracts" the beam. We see the same effect in the vertical collimators. This is a good experimental check of our theory.

The collimator kick leads to beam oscillations downstream of the collimator. The beam trajectory when a bottom jaw of a vertical collimator is moved closer to the beam is shown in Fig. 15. The solid line shows the vertical projection of the beam trajectory and the dotted line shows the horizontal projection.

In Fig. 15 we see the oscillations in the undulator region (last $100 \mathrm{~m}$ ), caused by the wake field effect from the collimator. It is interesting to note that we also get a horizontal oscillation when we move a vertical collimator. We have same unexplained coupling in this region.

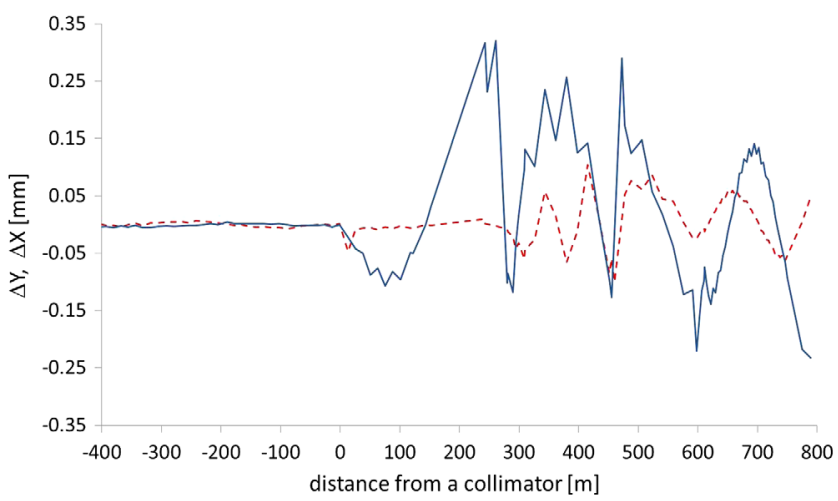

FIG. 15. Vertical (solid line) and horizontal (dotted line) projections of the beam trajectory when a bottom jaw of a vertical collimator is moved close to the beam. 


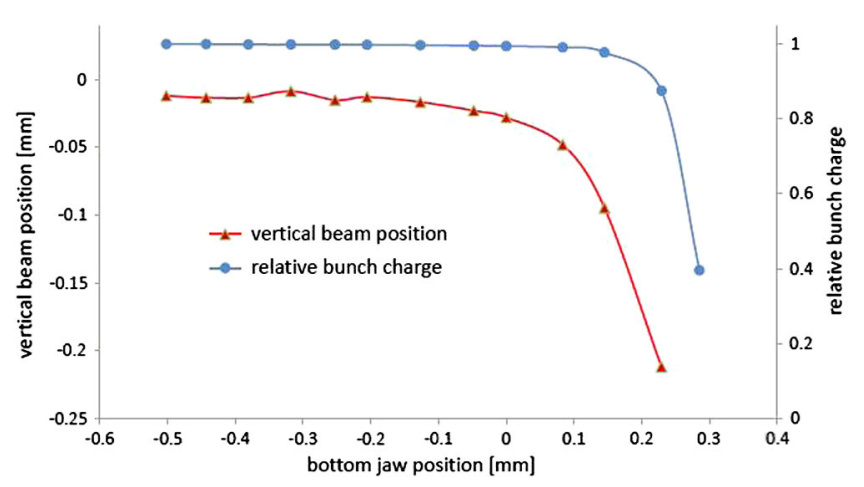

FIG. 16. Vertical beam position (line with triangles) and a relative bunch charge (line with circles) vs a collimator bottom jaw position.

To check the dependence of the averaged kick upon the collimator jaw position we use the BPM data at the place where the bunch gets the maximum displacement. As can be seen from Figs. 13-14 this region is approximately 60-80 m downstream the collimator. We have checked several collimators. Figure 16 shows a beam position and a relative bunch charge after a collimator as a function of a bottom jaw position.

We can see that the beam gets a kick before it touches the collimator jaw.

We compare our results with formula (14), which predicts a kick to be inversely proportional to the distance between a beam and a collimator jaw. To make an accurate comparison we approximate the measured data by our analytical prediction, optimizing the possible mistakes in measuring of a jaw edge position and a position of a BPM. The results are shown in Fig. 17 for the four jaws of a horizontal and a vertical collimator. In the measurement, we move each jaw to the beam keeping the other jaws far away from the beam. In these plots, the horizontal axis is a reverse position of a jaw. This makes the displacement a
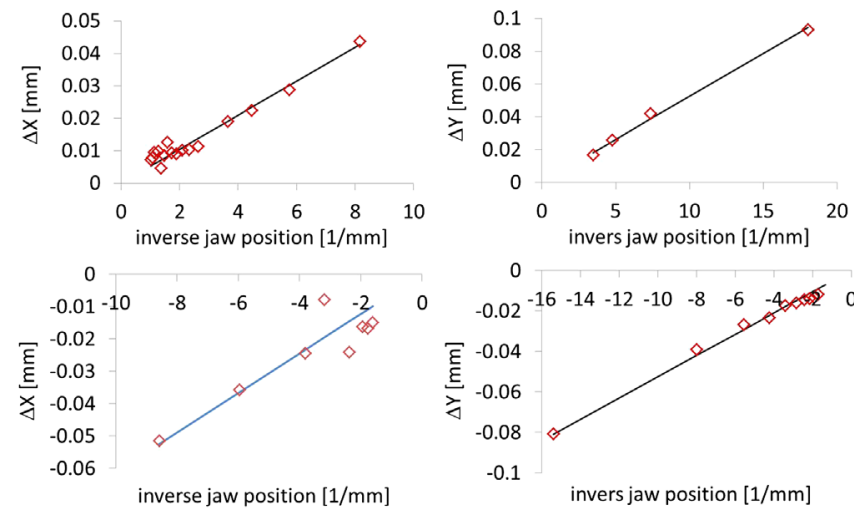

FIG. 17. Comparison of the measured displacement of a beam after a collimator (red diamonds) with the theoretical prediction (black solid line) for four jaws of the horizontal and vertical collimators. straight line. We see good agreement with our prediction for all jaws. However, we found that the calculated position of a collimator jaw is about $100 \mu \mathrm{m}$ closer to the beam in comparison with a jaw position, measured at the point when we lose $50 \%$ of the bunch charge. This can be explained by the fact that the beam has a nonzero transverse size. In future studies we will try to include a beam size to the formula (14). The error of the position of a BPM was found to be about $5-10 \mu \mathrm{m}$.

The special character of these wake fields is that the response reaction depends on the longitudinal position of the particles in the bunch. The "head" of the bunch is not deflected at all, but the "tail" gets the maximum deflection force. The transverse kick leads to the oscillations in the focusing system; the particles of the bunch will oscillate with different phases. This makes the bunch geometrically tilted. This tilt will also oscillate in the lattice. In reality the feedback system makes the head of the bunch oscillate too as it acts against the averaged kick. For the same reason the feedback system cannot compensate completely the kick from a collimator.

In the phase plane, the bunch tilt in the focusing system is rotating. Effectively the transverse emittance may be increased. We can make an estimate of this additional emittance assuming that the transverse beam size is twice the value of the displacement. Using a formula for the emittance

$$
\varepsilon_{i}=\frac{\left(\Delta x_{i}\right)^{2}}{\beta_{i}}, \quad i=x, y,
$$

and the values for the $\beta$ functions we calculate the effective emittance growth. We find that at some locations in the linac or LTU the effective (or projected) emittance can be comparable with the real beam emittance reaching more than $1 \mathrm{~mm}$ mrad. To check this we took an emittance measurement using an LCLS diagnostic in the LTU region [13]. The emittance measurement downstream of the collimator was taken using four wire scanners with a $45^{\circ}$ phase advance between them. The results are shown in Fig. 18 for three different positions of a collimator jaw. The left panels show the measured beam sizes in the vertical plane, and the right panels the respective normalized phase space ellipse. The dashed lines indicate the projection angle for each measurement. The projected emittance increases when a collimator jaw approaches the beam. We also can see a tilt of the bunch that rotates on the phase plane.

The bunch tilt oscillates in the focusing system of the FEL. This means that different particles of a bunch oscillate with different phases and that may disturb the coherent radiation in the FEL undulator. In this way the efficiency of the FEL performance may go down. We confirmed this prediction in the collected data. Usually the pulse energy of the x-ray beam describes the 
Emittance Scan on WIRE:LTU1:735

01-Apr-2014 03:56:22 Asymmetric
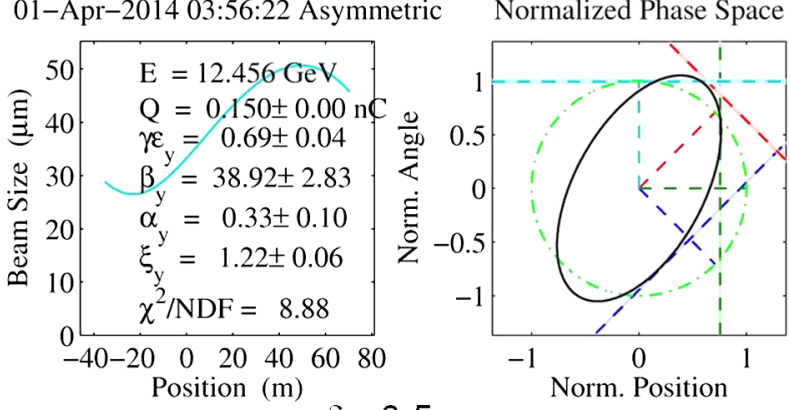

$\delta=-0.5 \mathrm{~mm}$
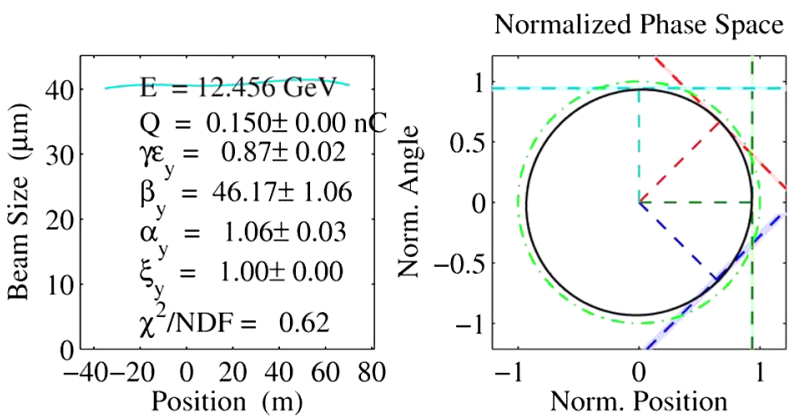

$\delta=+0.1 \mathrm{~mm}$
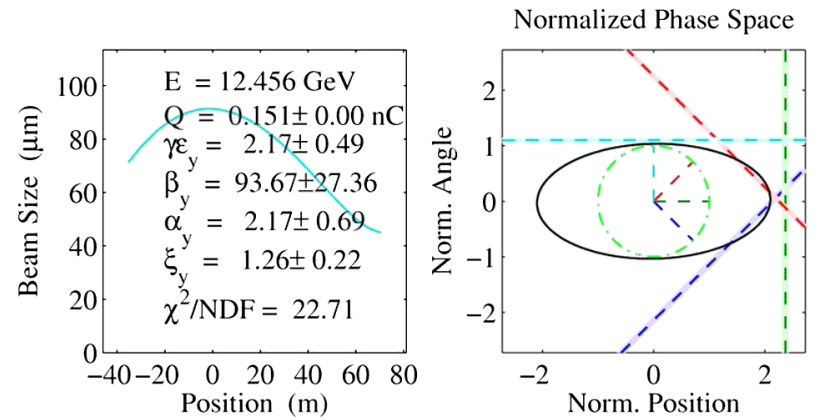

$\delta=+0.2 \mathrm{~mm}$

FIG. 18. Emittance measurements for different positions of the collimator jaw: $-0.5,+0.1,+0.2 \mathrm{~mm}$.

efficiency of the FEL. At the LCLS we use a gas detector to measure this parameter when we move a collimator jaw to the beam. The result of the measurement is shown at Fig. 19. We also plot in this figure the relative bunch charge and the projected emittance. We see a strong correlation between the growth of the projected emittance and the FEL pulse energy degradation. When a beam is close to a collimator jaw, a small change in the jaw position leads to a dramatic change in the x-ray production. We found that the pulse energy exponentially depends upon the particle loss. The pulse energy decreases by $50 \%$ when only $3 \%$ of the beam particles are absorbed by a collimator jaw.

We also see the rotation of the bunch on the energycoordinate phase plane using the X-band transverse deflector at the LCLS [14]. This deflector gives a linear kick along the bunch in the horizontal direction and particles

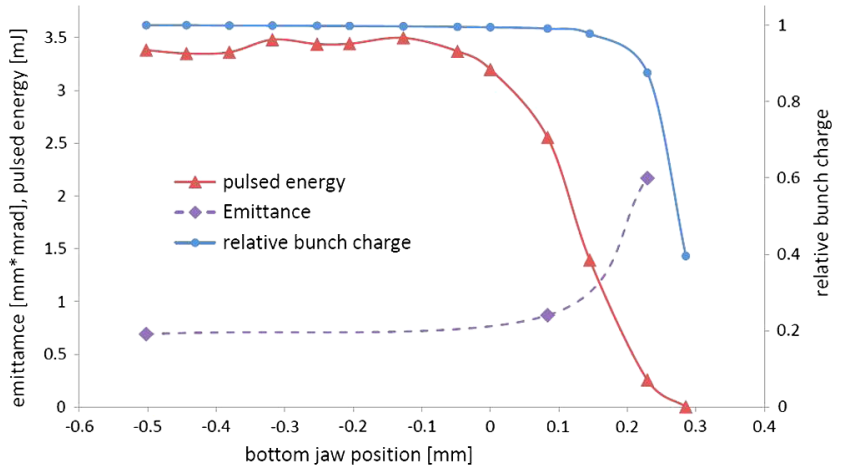

FIG. 19. FEL pulse energy (triangles), beam emittance (diamonds), and relative bunch charge (circles) vs the collimator jaw position.
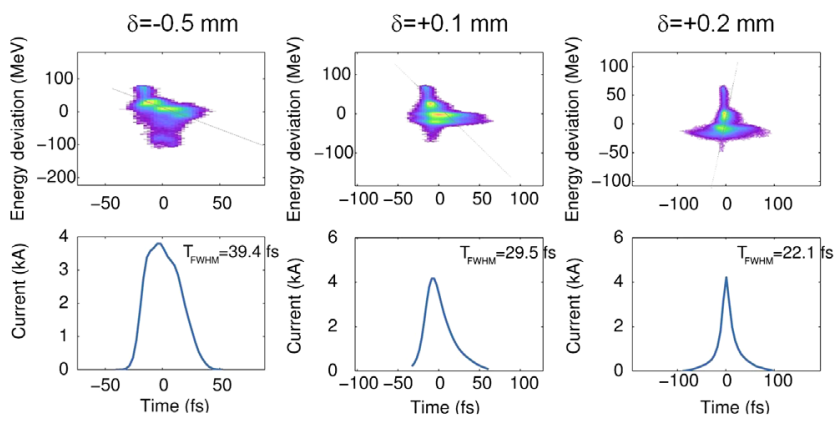

FIG. 20. Bunch images on the phase plane for different positions of the collimator jaw.

along the bunch take different horizontal positions. As the bunch goes to the screen after the vertical bending magnet, then the particles with different energies get different vertical positions. In the measurement we change the position of a jaw in a vertical collimator and then take images from the screen. A typical image of a bunch, which produces an X-ray pulse energy of $3 \mathrm{~mJ}$, is shown on the left plot of Fig. 20. As the collimator jaw comes closer to the beam (center and right plots at Fig. 20) the particles get transverse kicks opposite to the kick from the deflector. The energy spread also decreases as the x-ray production in the undulator degrades. The horizontal size of the beam also increases. We can explain this effect by the existence of the vertical-horizontal coupling in the LTU as we discussed before.

\section{SUMMARY}

We present a simple model and formulas for wake field effects from the SLAC linac collimators. We found this model to agree quite well with measurements. We found a strong effect from the collimators on performance of the FEL. A small amount of beam loss leads to dramatic degradation of the FEL performance. This study of the wake field effect from the collimators will be continued. 


\section{ACKNOWLEDGMENTS}

The authors would like to thank the LCLS physicists, operators, and engineers of the Accelerator Department for their help, especially A. Brachmann, J. Turner, D. Waltz, D. Kharakh, H. Loos, W. Colocho, and A. Fisher. This work was supported by Department of Energy Contract No. DOE-AC03-76SF00515.

[1] P. Emma et al., in Proceedings of the 10th European Particle Accelerator Conference (EPAC 2006), Edinburgh, Scotland (EPS-AG, Edinburgh, Scotland, 2006), MOPCH048.

[2] F.-J. Decker et al., in Proceeding of the XVIII International Linac Conference (LINAC96), Geneva, Switzerland, August 1996, http://accelconf.web.cern.ch/AccelConf/ 196/PAPERS/MOP34.PDF.

[3] P. Raimondi et al., Report No. SLAC-PUB-8552, 2000. Presented at 17th IEEE Particle Accelerator Conference (PAC 97), Vancouver, British Columbia, Canada, 1997 (unpublished).

[4] F. Zimmermann et al., in Proceedings of the European Particle Accelerator Conference (EPAC 1996), pp. 504-506 http://accelconf.web.cern.ch/AccelConf/e96/PAPERS/ TUPG/TUP099G.PDF.
[5] S. Molloy et al., in Proceedings of the 22nd Particle Accelerator Conference, PAC-2007, Albuquerque, NM (IEEE, New York, 2007), FRPMS074.

[6] P. Tenenbaum et al., Phys. Rev. ST Accel. Beams 10, 034401 (2007).

[7] V. Balakin and A. Novokhatski, in Proceedings of the 12th International Conference on High-Energy Accelerators (Fermilab, Batavia, Illinois, 1983), p. 117.

[8] D. Walz et al., Proceedings of the 1989 Particle Accelerator Conference, Chicago, IL (IEEE, New York, 1989); Report No. SLAC-PUB-4965.

[9] G. Dome, E. Gianfelice, L. Palumbo, V. G. Vaccaro, and L. Verolino, Il Nuovo Cimento 104A, 1241 (1991).

[10] A. Novokhatski, "On the estimation of the wake potential for an relativistic charge in an accelerating structure", Institute of Nuclear Physics, Novosibirsk, Preprint INP 88-39, 1988.

[11] A. Novokhatski, Report No. SLAC-PUB-11556, 2005 (unpublished).

[12] E. Gianfelice and L. Palumbo, IEEE Trans. Nucl. Sci. 37, 1084 (1990).

[13] H. Loos et al., in Proceedings of the 14th Workshop, BIW10, Santa Fe, NM, 2010.

[14] Y. Ding et al., Phys. Rev. ST Accel. Beams 14, 120701 (2011). 